\title{
An Adaptive Discrete Element Method for Physical Modeling of the Selective Laser Sintering Process
}

\author{
Arash Gobal ${ }^{*}$ and Bahram Ravani ${ }^{b}$ \\ Department of Mechanical and Aerospace Engineering, University of California Davis, Davis, CA \\ 95616, USA \\ aagobal@ucdavis.edu, bbravani@ucdavis.edu
}

Keywords: Selective laser sintering, discrete element method, adaptive simulation

\begin{abstract}
Selective Laser Sintering (SLS) has recently become one of the fastest growing additive manufacturing processes due to its capability of fabricating metal parts with high dimensional accuracy and surface quality. Physical modeling of selective laser sintering plays an important role in properly controlling the process parameters to achieve desired product properties. In this paper, we present a 3 dimensional, adaptive discrete element method for simulation of the SLS process on personal computers. The presented method models the laser-powder interaction at particle level, achieving high simulation accuracy while adaptively increasing the discrete element size as local temperatures drop inside the powder bed for improved efficiency. Numerical shape functions are developed for calculating individual particle temperatures at any point during the simulation. Results show that this physical model improves the runtime significantly in virtual simulation of SLS process without loss of simulation accuracy .
\end{abstract}

\section{Introduction}

Additive Manufacturing (AM), also known as rapid prototyping, rapid manufacturing, layered manufacturing and 3D printing, is an umbrella term often used to collectively define manufacturing processes that fabricate products directly from a 3D Computer Aided Design (CAD) model in a layer-wise fashion. It consists of a broad range of manufacturing technologies applied to various materials in different forms. Some of the current major AM technologies include Fused Deposition Modeling (FDM) for polymers, Selective Laser Sintering/Melting (SLS/SLM) for polymer, metallic and ceramic powders, Stereolithography (SLA) for liquid resins and Ultrasonic Consolidation (UC) for sheet metals [7].

The layer-wise fabrication approach of additive manufacturing, compared to the subtractive nature of traditional cutting processes, relaxes manufacturing constrains and allows optimal material distribution for optimizing the functionality of parts in order to achieve certain design criteria[1]. This also allows optimization of mechanical properties by enabling the manufacturing of parts with complex structures and lattices at no extra cost. Significant research has been done to develop methods that allow the designer to fully benefit from the design freedom of AM processes. These methods utilize shape, material, hierarchical and functional complexities provided by AM technologies to develop computational design methods for AM [28]. Parametric design [31], Topology optimization [2] and lattice structures [23] are among the methods that allow optimization of material distribution for AM products.

Advances in design methods and product quality for additive manufacturing has initiated industries such as aerospace, defense and automotive to show interest in AM technologies. As the major technology for additive manufacturing of metallic parts, powder bed fusion processes have experienced rapid growth recently. However, challenges in process modeling and control, predictive analysis of part properties and product qualification remain to be addressed for the powder bed based methods [26].

Selective laser sintering is one of the most popular powder bed fusion processes. Its capability of working with a wide variety of materials (polymer, ceramic, metallic and mixed powders), its ability 


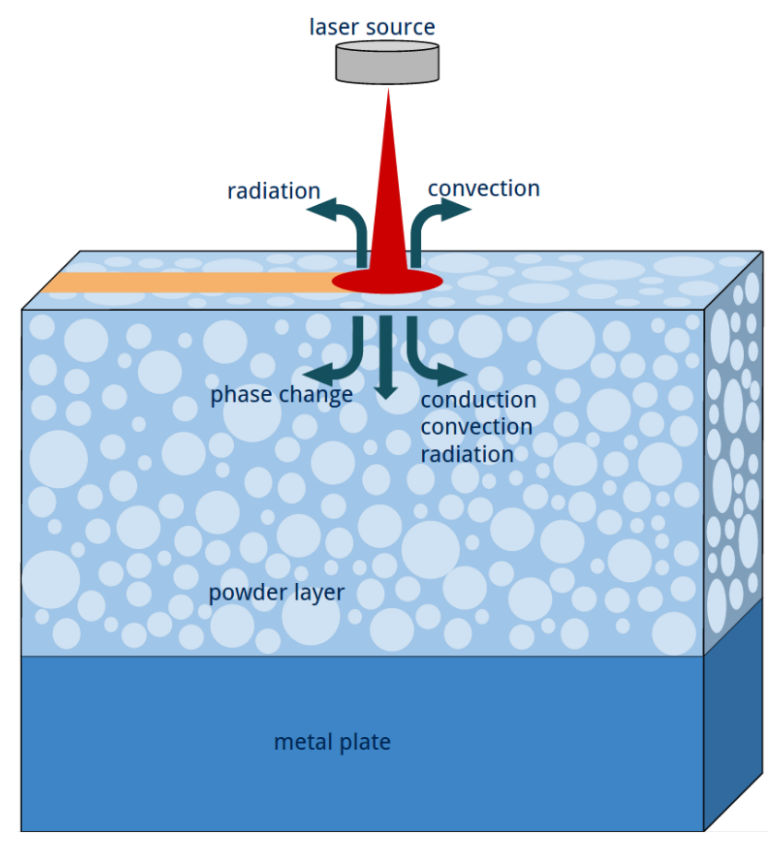

Fig. 1: Schematic of the SLS process

to create parts with desired porosity, and the dimensional quality of its products make it a popular candidate among other powder based AM technologies. During the SLS process a thin layer of powder (usually $50-100 \mu \mathrm{m}$ ) is deposited onto the powder bed. The surface of the powder is then scanned by a laser beam, heating and fusing powder particles together and creating the part cross section. Then, another layer of powder is deposited on top of the scanned layer and the scanning process is repeated until the part is fully fabricated. Next, the excess amounts of powder is removed and the final part is retrieved [21]. The main manufacturing process during the SLS process is therefore the selective fusion of powder particles as a result of heating by the laser beam. Fig. 1 shows a schematic of the SLS process.

Controlling the process parameters of the SLS process (laser power, laser beam speed, laser beam scanning pattern, preheating temperature, layer thickness etc.) is critical to creating a part with desired properties [6]. Powder bed temperature needs to be accurately controlled during the SLS process. Excessive heat could result in unwanted deformations, thermal stresses and poor dimensional accuracy. Where as insufficient heating would lead to incomplete powder fusion and poor mechanical properties of the final product [17]. A number of researchers have used experimental approaches for optimizing process parameters of the SLS process. For example, Chatterjee et al. [3] studied the application of statistical design of experiments to selective laser sintering of low carbon steel using a Nd:YAG laser. Moreover, Liao and Shie [15] conducted a Design of Experiments (DoE) method to achieve minimum porosity in SLS specimens. Although these studies predict the effect of process parameters on part properties, performing numerous experiments on SLS parts is expensive and time consuming. Therefore, the need for a general purpose simulation tool for physical modeling of the SLS process exists and is addressed in this paper.

During the past decade, a number of researchers have tried various methods for computer simulation of the SLS process. Due to the stochastic and porous nature of the powder bed, regular Finite Element Analysis (FEA) methods fail to provide acceptable solutions to the laser-powder interaction problem. Kolossov et al. [11] and Xing et al. [33] used fixed finite element models with temperature dependent properties and modeled the laser beam as a moving Gaussian heat input acting on top of the FE grid. Their models predict the peak temperature of the heated powder bed relatively accurately but fails to predict the transition of the particle level mictrostructure during the SLS process. Zeng et 
al. [34] and Pal et al. [22] developed a dynamic mesh method that enables the simulation of the melt pool geometry and temperature during the SLM process.

In all of the mentioned works, powdered material was treated as a continuum and its properties were averaged over a domain to generate FE stiffness and mass matrices for the simulations. Moreover, the transition in material properties as a result of selective heating and fusion of powder particles was also normalized over grids containing powder particles. Unless the finite element mesh length reaches the particle scale, continuum approximation of powdered material results in inaccuracies in temperature and microstructure modeling [5]. For these reasons, a number of researchers adopted the use of discrete element methods to perform process simulations of the SLS process. Zohdi [36] has developed discrete element methods for thermal simulation during the SLS process and also for heatinduced stress simulation for SLS parts. Based on these works, Ganeriwala and Zohdi [6] developed a coupled discrete element-finite difference model for SLS that allows modeling the interaction between powdered material and the continuous substrate. Moser et al. [18] used a discrete element ray-tracing method to predict the attenuation of the laser beam inside the powder bed. Kovaleva et al. [12] also developed a discrete element model for modeling the temperature and microstructure transition during the SLM process.

Compared to the finite element method, the discrete element method is capable of modeling the SLS process at the powder level, capturing the particle scale diffusion and transition of microstructure during the manufacturing process. However, because of the large number of particles involved in the fabrication of each part (usually in the order of millions), performing DEM simulations of the SLS process is extremely computationally expensive. For this reason, the authors have previously developed a multi-scale combined DEM/FEM method for rapid simulation of the SLS process [8].

In this paper, instead of a combined DEM/FEM method, an adaptive discrete element model for thermo-mechanical modeling of the SLS process is developed, validated and implemented. The adaptive method increases the size of elements in the DEM based on their sintering potential (particle temperature) and their location relative to the moving laser heat source. In this manner, the proposed physical modeling method is capable of carrying out the simulation at a reasonable speed on a personal computer. Fusion of powder particles during laser-powder interaction is also modeled to ease predicting the microstructure and mechanical properties of the SLS product for future works. Simulation results are validated against published works and the improvement in runtime compared to the particle-level discrete element model is demonstrated.

\section{The adaptive discrete element method}

In this section, the development of the adaptive discrete element model for physical modeling of the SLS process is presented. The DEM has been employed for the simulation of powder packing inside the bed, heat transfer between powder particles and fusion of particles under the influence of a moving heat source (laser beam). A number of simplifying assumptions have been made to make the model run fast without losing much of the computational accuracy which are explained in each relevant subsection. Moreover, in order to reduce computation time, an adaptive method has been developed which will adjust particle sizes with respect to their temperature and distance from the moving heat source. This approach builds upon the multi-scale model developed previously by authors [8].

Packing generation. The first step in performing computer simulation of the SLS process is generating a computer model for packing of powder particles which resembles the real properties of the powder bed. These properties include powder size distribution, packing density (the ratio of total volume of powders inside the packed bed to the total volume of the bed) and average coordination number (average number of neighbors of each particle inside the packing). In order to meet requirements for all these properties, a Monte-Carlo simulation model developed by He et al. [10] was used in this study to generate the powder bed with the desired characteristics. This model consists of the following steps: 
1. An array of $n$ particles with the desired size distribution is generated. Powders used for SLS applications often follow a log-normal size distribution. In this case, the probability density function of particle radius $r$ is:

$$
f(r)=\frac{1}{\sqrt{2 \pi} \sigma r} e^{-\left(\log r-\log r_{0}\right)^{2} / 2 \sigma^{2}}
$$

where logr is the natural logarithm of $r$ and $r_{0}$ and $\sigma$ are the mean and standard deviation of $\log [10]$.

2. Size of the bounding box is determined using the following formula (considering a cubic bed):

$$
L_{0}=\left[\frac{1}{\phi_{0}} \sum_{i=1}^{n} \frac{4}{3} \pi r_{i}^{3}\right]^{1 / 3}
$$

where $\phi_{0}$ is the desired packing density of the powder bed, $r_{i}$ is the radius of the $i$ th particle and $L_{0}$ is the side length of the bed.

3. Particles are randomly placed inside the calculated volume regardless of overlaps,

4. For each particle $i$, all the overlapping particles $j$ are obtained using the following formula:

$$
o_{i j}=\frac{r_{i}+r_{j}-d_{i j}}{r_{i}+r_{j}}
$$

where $o_{i j}$ is the calculated overlap between particles $i$ and $j$ and $d_{i j}$ is the distance between their centers. For each pair of particles $i$ and $j$, a positive $o_{i j}$ shows that the two particles overlap each other.

5. For each particle $i$ inside the packed bed that is overlapped by at least one particle, a new location can be found using the following formula:

$$
{\overrightarrow{R_{i}}}^{\prime}=\frac{1}{n_{i}} \sum_{j=1}^{n_{i}} \overrightarrow{R_{i j}}
$$

where $\vec{R}_{i}^{\prime}$ is the vector showing the new location of particle $i, n_{i}$ is the total number of particles overlapping particle $i$, and $\overrightarrow{R_{i j}}{ }^{\prime}$ is the rearrangement imposed to decrease the overlap created by the $j$ th overlapping particle and is calculated as:

$$
\overrightarrow{R_{i j}}=\overrightarrow{R_{j}}+\left(\overrightarrow{R_{i}}-\overrightarrow{R_{j}}\right) \frac{r_{i}+r_{j}}{d_{i j}}
$$

6. steps 4 and 5 are repeated until either the maximum overlap inside the powder bed drops below a specified treshold or a maximum number of iterations is reached (in cases where the defined packing fraction is unrealistic).

This algorithm is capable of creating realistic models of packed powder beds. However, as the number of particles inside the system increases, the model faces significant increase in runtime. The reason is that during step 4 of the algorithm, all particles inside the packing are tested for finding overlapping particles. This has a considerable contribution to the increase in simulation runtime. In order to reduce the runtime for simulation of packing of large powder beds, the bed is divided into a number of grids each holding a relatively small number of particles inside them. This way, the search for overlapping particles is only performed within neighboring grids. This approach is able to improve 


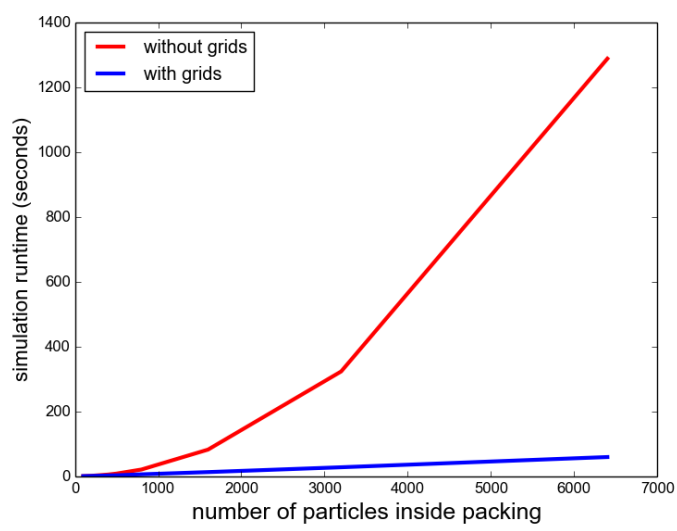

Fig. 2: Comparison of runtime for powder packing generation with and without using grids

the algorithm runtime. Fig. 2 illustrates the comparison of runtime between models with and without the grids.

Thermal simulation. As the main manufacturing mechanism in the SLS process is heating and fusion of particles under the influence of a moving laser beam, accurate simulation of heat transfer between powder particles is an integral part of this physical modeling method. All three major mechanisms of heat transfer (conduction, convection and radiation) are present during the heating and fusion of powder particles. The general governing equation for heat transfer during the SLS process is therefore [6]:

$$
\int_{\partial \omega} \text { Q.n } d A+\int_{\omega} H d V=\int_{\omega} \rho c \dot{T} d V
$$

where $\mathbf{Q}$ represents particle-particle heat transfer, $H$ accounts for the laser beam heat input, $\rho$ is the material density, $c$ is the specific heat of the material, $T$ is the temperature and $\omega$ represents the domain of interest (one spherical particle in this case) [6].

Certain assumptions can result in significantly reducing the computational complexity of the problem while maintaining high simulation accuracy. One of them is the assumption of uniform temperature inside each powder particle during the simulation. Calculation of the dimensionless Biot number (ratio of internal to external thermal resistance of a solid body) helps determining the validity of this assumption [6]:

$$
B i=\frac{h s}{k}
$$

where $h$ is the average heat transfer coefficient on the unit surface, $k$ is the thermal conductivity of the solid material and $s$ is the characteristic length of the body, which is commonly defined as the volume of the body divided by its surface area. According to Ganeriwala and Zohdi [6], for low Biot numbers (below 0.1) particles can be safely assumed to have uniform temperatures. Under this assumption, the heat transfer equation can be discretized for each particle $i$ as:

$$
Q_{i}+H_{i}=m_{i} c_{i} \dot{T}_{i}
$$

This equation allows modeling the powder bed as a collection of $n$ nodes that are connected together with thermal resistances (Fig. 3). $Q_{i}$ in Eq. 8 is the total heat transfer between particle $i$ and its surrounding particles and is defined as:

$$
Q_{i}=Q_{i}^{c o n d}+Q_{i}^{c o n v}+Q_{i}^{r a d}
$$




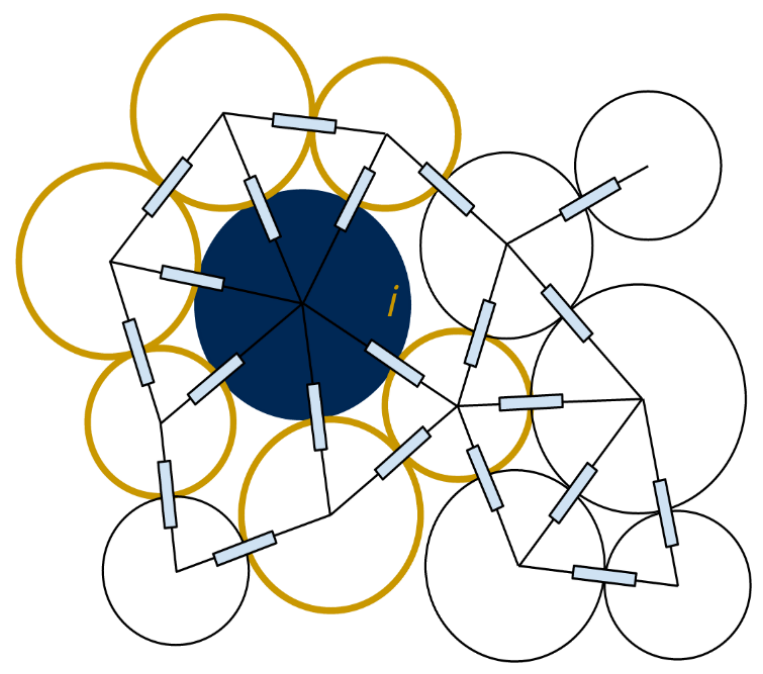

Fig. 3: 2 dimensional schematic of the powder bed model used in this paper

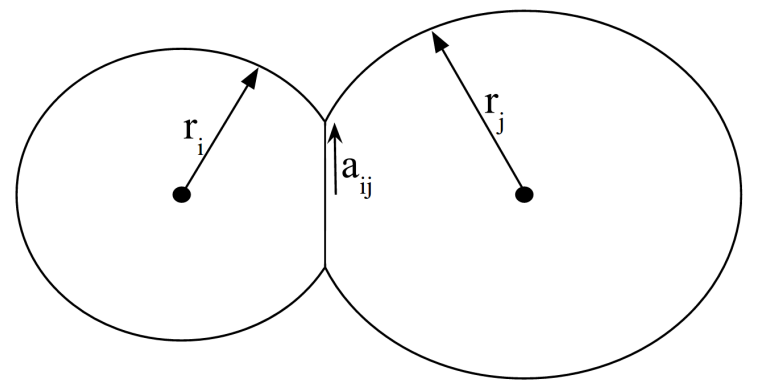

Fig. 4: Model of the Hertzian contact between two particles

$Q_{i}^{\text {cond }}$ is the total heat input to particle $i$ from its surrounding particles due to conduction and can be formulated as [9]

$$
Q_{i}=\sum_{j=1}^{m} K_{i j}\left(T_{j}-T_{i}\right)
$$

where $K_{i j}$ is the heat conduction coefficient between neighboring particles $i$ and $j$ and $m$ is the number of particles that are in contact with particle $i$. According to Moser et al. [19], heat conduction between two contacting solid particles occurs both through the contact area and the thin gas layer around the contact area. Therefore, $K_{i j}$ can be written as

$$
K_{i j}=K_{i j}^{\text {particle-particle }}+K_{i j}^{\text {particle-gas-particle }}
$$

- The coefficient of heat conduction through the contact points between particles has been developed by Liang and $\mathrm{Li}[14]$ as:

$$
K_{i j}^{\text {particle-particle }}=2 \lambda_{p} a_{i j}
$$

where $\lambda_{p}$ is the heat conduction coefficient of the solid material and $a_{i j}$ is the radius of the contact area between two particles (assuming spherical, elastic, smooth particles) as shown in Fig. 4. Radius of the contact area is calculated using the Hertzian contact theory between two elastic spheres $i$ and $j$ of radius $r_{i}$ and $r_{j}$ pressed in contact with force $F$ [32]:

$$
a_{i j}=\sqrt[3]{\frac{3 F r_{i j}}{4 E^{*}}}
$$




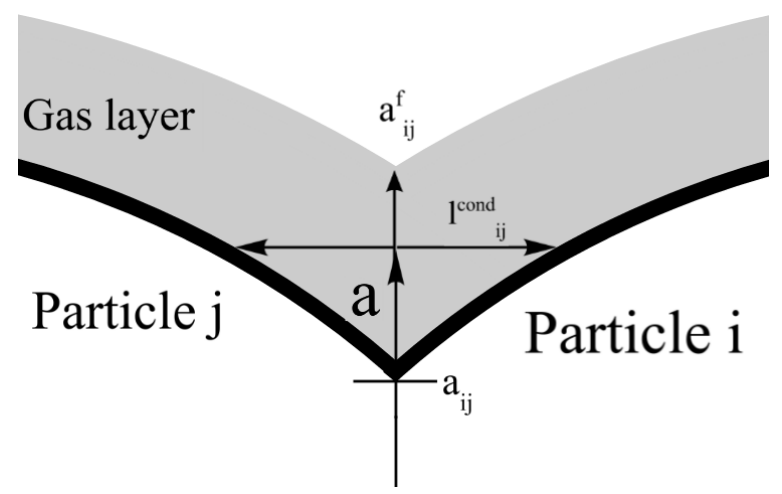

Fig. 5: Model of particle-gas-particle heat conduction used by Moser et al. [19]

where $E^{*}$ is the equivalent Young's modulus for contact defined as

$$
\frac{1}{E^{*}}=\frac{1-\nu_{i}^{2}}{E_{i}}+\frac{1-\nu_{j}^{2}}{E_{j}}
$$

and $r_{i j}$ is the reduced radius of the curvature and is related to radii of contacting particles using the following formula:

$$
\frac{1}{r_{i j}}=\frac{1}{r_{i}}+\frac{1}{r_{j}}
$$

Calculating the contact area radius using equation 13 requires knowing the interparticle contact force $(F)$ at all contact locations. $F$ is therefore obtained based on the location of the contact area and the average number of particles on top of it.

It should be noted that this model assumes particles to be perfectly spherical, smooth and elastic, which is usually not the case for particles used in SLS processes. However, this assumption proves to generate valid results in heat transfer analysis of packed beds [14]. Moreover, when particles are heated up and sintered together, this model can no longer predict the heat conduction coefficients for neighboring particles accurately and therefore needs to be updated.

- Heat conduction also occurs through the gas layer around the particles' contact areas. It should be noted that this type of heat transfer only occurs when the gas layer is relatively thin and it only involves heat conduction through the thin gas layer. According to Moser et al. [19], conduction coefficient of the gas layer is calculated using the following formula:

$$
\begin{gathered}
K_{i j}^{\text {particle-gas-particle }}=K_{g a s} \int_{a_{i j}}^{a_{i j}^{f}} \frac{2 \pi a}{l_{i j}^{c o n d}} d a \\
l_{i j}^{\text {cond }}=l_{i j}-\left(\sqrt{R_{i}^{2}-r^{2}}+\sqrt{R_{j}^{2}-r^{2}}\right)
\end{gathered}
$$

Where $l_{i j}^{\text {cond }}$ is the length of the conductive gas layer between particles $i$ and $j$ at a given $a$ value, $l_{i j}$ is the distance between the centers of particles, and $a_{i j}^{f}$ is the radius of the fluid layer surrounding the contact area that accounts for the heat conduction (Fig. 5). According to Musser [20], $a_{i j}^{f}$ can be chosen consistently as $0.2 r_{i j}$.

Heat transfer between the powder particles and the underlying substrate is also calculated using the particle-particle heat conduction formula. 
$Q_{i}^{c o n v}$ is the convective heat transfer between powder particles and the surrounding gas and is formulated using the following equation:

$$
Q_{i}^{c o n v}=h_{\text {conv }}\left(T_{e n v}-T_{i}\right) A_{i}
$$

Where $h_{c o n v}$ is the convection coefficient and $A_{i}$ is the surface area of particle $i$ [6]. In this study, convective heat transfer is only calculated for powders on the top layer of the powder bed. The reason is that the relatively small inter-particle distances inside a packed powder bed prevents heat convection to occur inside the packed bed [25].

$Q_{i}^{r a d}$ is the heat transfer between each particle and its surrounding through radiation. For highenergy particles inside the domain, radiation heat transfer could also play a role in temperature calculations. Radiation heat transfer is formulated as follows:

$$
Q_{i}^{r a d}=\epsilon \sigma_{S B}\left(T_{e n v}^{4}-T_{i}^{4}\right) A_{i}
$$

where $\epsilon$ is material emmisivity and $\sigma_{S B}$ is the Stefan-Boltzmann constant $\left(\sigma_{S B}=5.67 \times 10^{-8} \mathrm{~W} /\right.$ $\left.m^{2} K^{4}\right)$.

Modeling the moving heat source. It has been shown that the moving laser beam can be modeled as a moving Gaussian heat source acting on top of the powder bed. With this assumption, the $H_{i}$ term in equation (8) can be written as

$$
H_{i}=\alpha I_{0} \beta \exp \left(-2 \frac{\left(x-x_{i}\right)^{2}+\left(y-y_{i}\right)^{2}}{\omega^{2}}-\beta z\right) A_{i}
$$

Where $\alpha$ is the optical absorptivity of the powder bed, $\beta$ is the extinction coefficient, $\omega$ is the laser beam characteristic radius where the laser intensity $(I)$ drops to $1 / e$ of its peak value $(\omega=D / 4.292$, $D$ being the laser beam diameter), $x$ and $y$ are coordinates of the center of the laser beam and $I_{0}=\frac{2 P}{\pi \omega^{2}}$ is the peak intensity.

Because of the porous nature of granular materials, the laser is able to penetrate up to several particle diameters' deep into the powder bed [11]. In this work, the results of the Monte Carlo raytracing algorithm developed by Moser et al. [18] is used to obtain the optical absorptivity and extinction coefficients of the powder bed. In this method, a large number of rays are simulated to be fired into the powder bed and are allowed to reflect from powder particles until their intensity drops below 1 percent of the original value. By calculating the amount of energy absorbed at each certain depth of the powder bed, empirical values for extinction coefficient and absorptivity of powder packings can be obtained. Absorption rate of the material as well as particle size distribution and packing density of the powder bed are major factors determining the optical absorption and extinction coefficients of the powder bed.

Time step calculation. Although several high order numerical time integration schemes have previously been developed for DEM and DEM/FEM simulations, the study of stability, accuracy and computational efficiency of these algorithms show that none of the developed schemes are unconditionally stable and their accuracy can all be improved by choosing smaller time steps regardless of the chosen method. However, lower-order schemes are shown to be faster than more complex, high-order integration schemes [29]. Therefore, in this work we use an Euler explicit integration method to update the particle temperatures at each time step [30]:

$$
T_{i}^{t+\Delta t}=T_{i}^{t}+\frac{Q_{i}^{t}}{m_{i}} \Delta t
$$

For stability of the numerical integration, we need to choose the simulation time step to be less than the critical time step $\left(\Delta t<<\Delta t_{\text {critical }}\right)$. The critical time step is obtained from:

$$
\Delta t_{\text {critical }}=\sqrt{\frac{m}{K}}
$$


where $m$ is the particle mass and $K$ is the thermal stiffness coefficient for the 1 DoF particle system.

Simulation of particle fusion. The main sintering mechanism simulated in this study is the Solid State Sintering (SSS). Solid state sintering occurs in temperatures between $T_{\text {melt }} / 2$ and $T_{\text {melt }}$ of the solid material and the major binding mechanism during this process is volume diffusion [13]. During the simulation, when temperature of a particle couple is below $T_{\text {melt }} / 2$, heat transfer between particles is calculated using the model described earlier. However, when the temperature passes $T_{\text {melt }} / 2$, we need to have an additional model for simulation of the volume diffusion during the sintering process [27].

Effective physical modeling of powder sintering requires introduction of a formula for simulation of the high temperature volume diffusion between powder particles which later results in shrinkage of SLS products. Using the discrete element model as a preferred framework for simulation of the sintering process, particle diffusion can be described using the formula for the relative axial velocity of two neighboring particles during sintering developed by Parhami and McMeeking [24] for solid state sintering. The models is described by the following equations:

$$
\begin{gathered}
V_{n}=\frac{8 \mathscr{D}_{b}}{r^{2}} \sigma-\frac{8 \mathscr{D}_{b} \gamma_{s}}{r^{4}}\left[4 R\left(1-\cos \frac{\Psi}{2}\right)+r \sin \frac{\Psi}{2}\right] \\
\dot{r}=-\frac{8 R \mathscr{D}_{b}}{r^{3}} \sigma+\frac{8 R \mathscr{D}_{b} \gamma_{s}}{r^{5}}\left[4 R\left(1-\cos \frac{\Psi}{2}\right)+r \sin \frac{\Psi}{2}\right]
\end{gathered}
$$

where $a$ is the radius of the contact area between the two particles, $R$ is the particle radius, $\gamma_{s}$ is the surface energy per unit area, $\Psi$ is the dihedral angle at the neck formed at the particle contact point, $V_{n}=V_{n}^{1}-V_{n}^{2}$ is the relative axial velocity of one particle with respect to the other and $\mathscr{D}_{b}$ is the effective grain boundary diffusivity defined as:

$$
\mathscr{D}_{b}=\frac{D_{g} \delta_{g} \Omega}{k T}
$$

where $D_{g}$ is the diffusion coefficient for vacancy transport in the grain boundary with thickness $\delta_{g}, \Omega$ is the atomic volume and $k$ is the Boltzmann constant. Moreover,

$$
\sigma=\frac{F_{n}^{(1)}}{\pi r^{2}}=-\frac{F_{n}^{(2)}}{\pi r^{2}}
$$

is the normal stress applied on the particle grain boundaries.

Under the above model for stress-free sintering, the contact radius between two particles during sintering grows until the sum of grain boundary energy and surface energy of the particles reaches a local minimum, where any perturbation would increase the total energy. The size of the contact radius at this equilibrium point is calculated using the following formula [24]:

$$
a_{e q}=R \sin \frac{\Psi}{2}
$$

It must be noted that Eqs. 23-27 were developed for mono-disperse particles. However, Martin et al. showed that by replacing $R$ with the equivalent radius of the two contacting particles $R^{*}=$ $R_{1} R_{2} /\left(R_{1}+R_{2}\right)$ the above model can be used for any particle size distribution [16].

Hierarchical DEM. Adaptive hierarchical DEM is proposed here to speed up the simulation process. Although particle-scale heat transfer analysis is essential for an accurate simulation of the SLS process. Studies have shown that the effect of individual particle contact areas can be effectively averaged over a large number of particles in areas with moderate temperature gradients [14]. The hierarchical adaptive DEM replaces particle-level elements with larger discrete elements if their temperature falls below a specified amount. The convection, conduction and radiation properties of the 


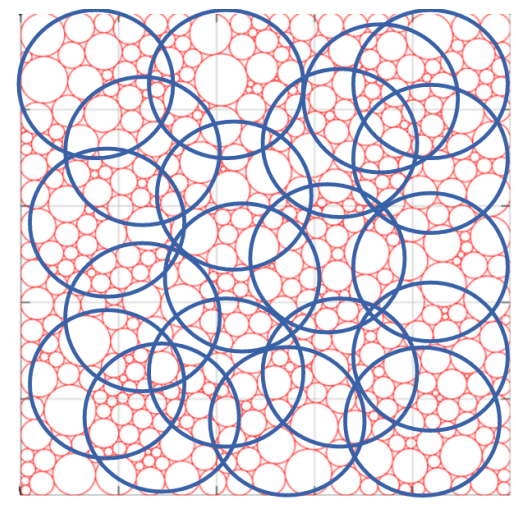

Fig. 6: 2 dimensional schematic of the coarse and fine scale DEM

large element are then calculated based on the particle-level elements that it contains. A numerical shape function is also defined for interpolation of element temperatures in order to find particle temperatures at any point during the simulation. Fig. 6 shows a $2 \mathrm{~d}$ illustration of the coarse vs. fine scale discrete element model.

In this method, the cut-off temperature for shifting the simulation to coarse DEM elements was chosen to be $T_{\text {melt }} / 2$. The reason is to maintain the model's capability in performing accurate particlelevel sintering simulations as the actual sintering starts at $T_{\text {melt }} / 2$. As powder particles rapidly lose their thermal energy, the coarse elements will be capable of replacing particle level elements and increase computation speed. Moreover, the coarse element size is chosen as the maximum value that is capable of reproducing particle-level temperatures by interpolation using element shape functions.

Development of the numerical shape functions for the adaptive discrete element method in this paper is based on the work of Zhang et al. on development of a multi-scale method for thermal conduction simulation in granular materials [35]. The discrete treatment of the heat transfer problem assumes all heat transfer to occur in a network of nodes (powder particles) connected with thermal resistance elements (particle contact areas). The coarse elements also follow the same rule. Each particle resides in at least one coarse element. Assuming there are $N$ particles in a sample powder bed, the temperature field can be represented by the following array:

$$
\mathbf{T}=\left\{T^{1}, T^{2}, T^{3}, \ldots, T^{N}\right\}^{T}
$$

Knowing that each particle belongs to at least one coarse element, $T$ can be described as:

$$
\mathbf{T}=\boldsymbol{\Phi} \mathbf{T}_{\mathbf{C}}
$$

where $\mathbf{T}_{\mathbf{C}}=\left\{T_{C}^{1}, T_{C}^{2}, T_{C}^{3}, \ldots, T_{C}^{k}\right\}$ is the array of coarse element temperatures and $k$ is the total number of coarse elements inside the powder bed sample. Moreover, $\boldsymbol{\Phi}=\left\{\Phi_{1}, \Phi_{2}, \Phi_{3}, \ldots, \Phi_{N}\right\}^{T}$ is the numerical shape function matrix where:

$$
\Phi_{i}=\left\{\phi_{i}^{1}, \phi_{i}^{2}, \phi_{i}^{3}, \ldots, \phi_{i}^{k}\right\}
$$

is the shape function array for each particle with respect to its neighbor elements.

To obtain the value of the shape functions, the steady state result of the boundary value problem at each coarse element inside the packing is determined. The results were validated against the shape function criteria and were shown to follow them closely.

\section{Model Validation}

Validation of the powder packing method and the use of discrete element method for heat transfer simulations of granular materials has been previously performed by authors in [8]. In this section we 
study the validation of the heat deposition model by studying the heat affected zone (HAZ) and the accuracy of the proposed adaptive DEM by performing shape function validation calculations and solving a sample heat transfer problem.

Laser beam radiation. Validation of the Gaussian laser beam model, its penetration into the powder bed, and the laser-powder interactions can be best studied by running a numerical simulation of the SLS process based on known material properties and process parameters. For this purpose, a realistic powder bed was generated based on the physical properties of stainless steel powders from Dayal and Gambaryan-Roisman [4] as shown in Table 1. This table also includes the parameters obtained from Parhami and McMeeking [24] for simulation of the powder fusion mechanism. Moreover, the laser beam was modeled as a Gaussian heat input on the top surface of the powder bed. Laser extinction coefficient inside the powder bed was obtained from Moser et al. [18]. Process parameters of the simulation are shown in Table 2.

Table 1: Properties of the powder bed [6][4][24].

\begin{tabular}{lc}
\hline Property & value \\
\hline Packing density & $60 \%$ \\
Particle diameter $r,[\mu \mathrm{m}]$ & $25,50,90$ \\
Density $\rho_{s},\left[\mathrm{~kg} / \mathrm{m}^{3}\right]$ & 7800 \\
Melting temperature $T_{m},\left[{ }^{\circ} \mathrm{C}\right]$ & 1500 \\
Specific heat $C,[\mathrm{~J} / \mathrm{kgK}]$ & 600 \\
Solid thermal conductivity $\lambda_{s},[\mathrm{~W} / \mathrm{mK}]$ & 40 \\
Young's modulus $E,[\mathrm{GPa}]$ & 210 \\
Poisson's ratio $\nu$ & 0.28 \\
Absorption coefficient $K_{a b}$ & 0.3 \\
Surface energy $\gamma_{s},\left[\mathrm{~J} / \mathrm{m}^{2}\right]$ & 1 \\
Dihedral angle $\Psi,\left[{ }^{\circ}\right]$ & 146 \\
Atomic volume $\Omega,\left[\mathrm{m}^{3}\right]$ & $1.18 \times 10^{-29}$ \\
Diffusion coefficient $D_{g} \delta_{g},\left[\mathrm{~m}^{3} / \mathrm{s}\right]$ & $3.832 \times 10^{-19}$ \\
Attenuation coefficient $\beta,[1 / \mathrm{mm}]$ & 0.023 \\
\hline
\end{tabular}

Table 2: Process parameters [4].

\begin{tabular}{lc}
\hline parameters & value \\
\hline Laser power $P,[W]$ & 50 \\
Laser beam speed $v,[\mathrm{~m} / \mathrm{s}]$ & 1 \\
Laser spot radius $\omega_{l},[\mathrm{~m}]$ & $1.4 * 10^{-4}$ \\
\hline
\end{tabular}

As can be seen in table 1, validation of the laser sintering model is done for three different particle size distributions. The packing density of the powder bed is kept at $60 \%$, which follows the realistic packing density for SLS applications [4].

The sample powder bed generated for this simulation consists of about 8000 mono-disperse powder particles with the diameter of $25 \mu \mathrm{m}$ with an initial preheat temperature of $373 \mathrm{~K}$. The bed dimensions are $2 \times 0.4 \times 0.1$ millimeters which describes a powder bed large enough for performing realistic simulations. Because of the fast laser scanning speed relative to the powder bed size, a constant time step of $5 \mu \mathrm{s}$ was chosen to allow accurate simulation of heating and cooling of the particles, This value falls well below the critical time step required for simulation. The Gaussian laser beam is then modeled to scan a line on top of the powder bed and transient temperatures during the process are recorded.

Fig. 7 shows visualization of sintering a line on the top layer of the powder bed. It shows that the general shape of the heat affected zone and heat transfer through the powder bed match with results 


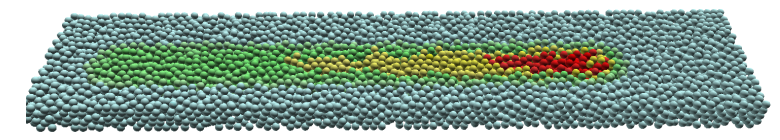

Fig. 7: Visualization of the SLS process on the top layer of the powder bed

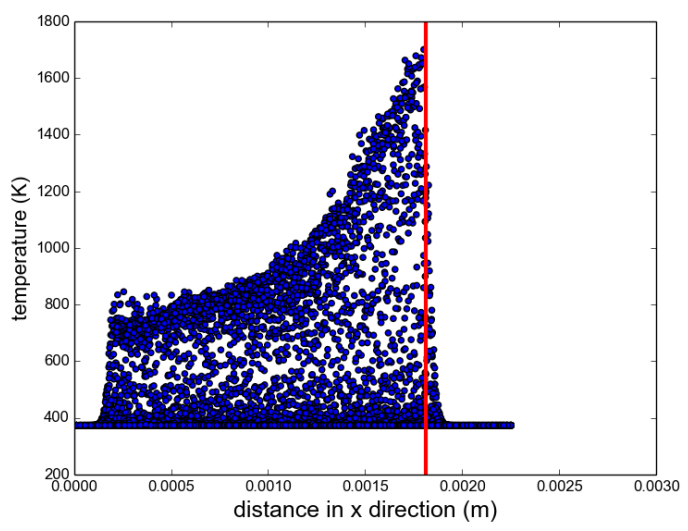

Fig. 8: Particle temperatures during the SLS process

previously reported in studies such as [6] and [4]. Also, Fig. 8 shows the temperatures of each powder particle at a point during sintering (the red line shows the location of the laser beam at that point in time). The trend of this figure and the numerical values of the particle temperatures compared with results provided by Kolossov et al. [11] and Xing et al. [33] prove the credibility of our model for use in physical modeling of the SLS process.

It must be noted that an actual numerical comparison with experimental results is not possible at the moment as the authors did not have access to all the required process parameters of published experiments for reproducing their results.

Adaptive Hierarchical DEM. The first step of using the adaptive DEM physical modeling and simulation tool is validation of its numerical shape function used for interpolation. The numerical shape function was developed by solving the steady state boundary value problem on one random node and fitting a polynomial function to the steady state solution. The Kronecker delta property of the shape function is then forced on the polynomial equation.

The calculated numerical shape functions satisfies the Kronecker delta property and they are capable of interpolating the field variable (particle temperatures) between nodes. Fig. 9 shows the solution of a steady state heat transfer problem through a powder bed using both powder level and interpolated values using the developed numerical shape functions from the coarse DEM solution. It can be seen that the shape functions are capable of relative accurate interpolating the temperature for each particle inside the powder bed. It can therefore be concluded that this method shows potential for use in adaptive DEM simulations.

It must also be noted that although numerical shape functions perform adequately in interpolation of temperature at particle locations, They are not exactly replicating the results of a particle level simulation in order to save computation time. However, our results shows that the error associated with this approximation are relatively small and doesn't contribute to simulation inaccuracy.

\section{Results}

We used the adaptive discrete element method for physical modeling and simulation of the selective laser sintering process of stainless steel powders. The switch between coarse and fine DEM was performed based on the maximum powder temperature in the region as well as the particle's proximity 


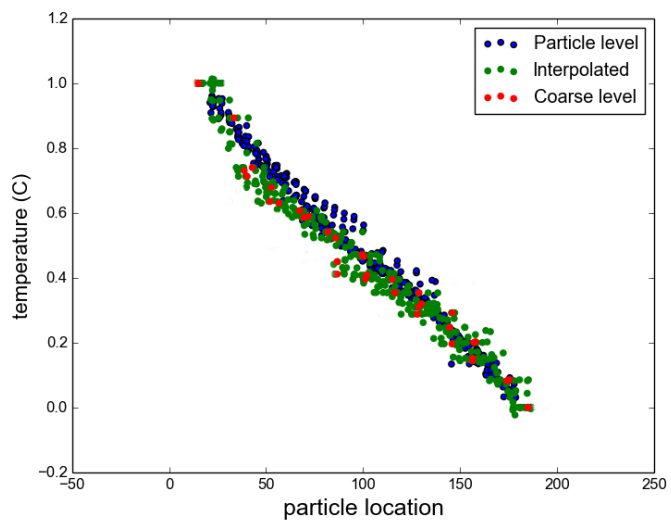

Fig. 9: Steady state heat transfer in powder bed using the multiscale DEM

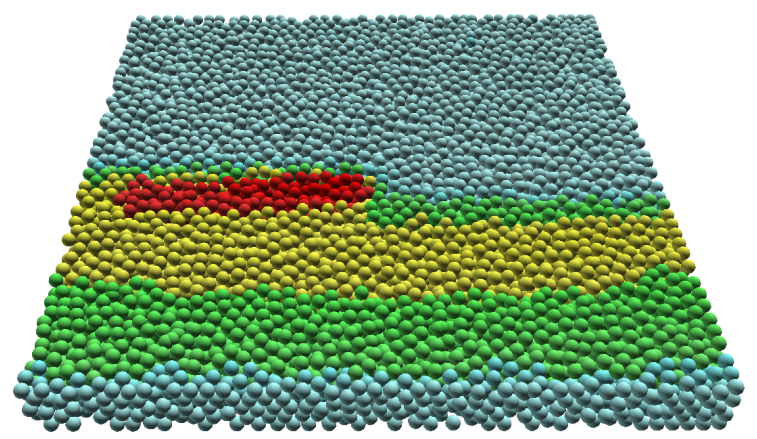

Fig. 10: Simulation of SLS on a $1 \mathrm{~mm}$ by $1 \mathrm{~mm}$ layer of powder particles

to the laser beam. As powder particles reach the sintering temperature $\left(T_{\text {melt }} / 2\right)$, the fine powders replace coarse discrete particles in order to increase the accuracy of the simulation. Moreover, regions of the bed that are next to be scanned by the laser beam are also switched to find particles in order to increase the accuracy of the laser-powder interaction simulation.

Fig. 10 shows the temperature distribution on a $1 \mathrm{~mm} \times 1 \mathrm{~mm}$ powder bed during the SLS process. The diameter of the mono-disperse powders used in this simulation is $25 \mu \mathrm{m}$ and they are heated with a $25 \mathrm{~W}$ laser beam with a $1 \mathrm{~m} / \mathrm{s}$ speed. Peak temperature during this process reaches about $1500 \mathrm{~K}$.

The described simulation was performed using both traditional DEM and the developed adaptive DEM process. In this setting, the adaptive DEM process was capable of improving the heat transfer segment of the simulation by a factor of 30 . Including the preprocessing time (time required for the powder packing generation), the total simulation runtime was improved by a factor of 4.25 .

\section{Conclusions}

A new adaptive discrete element method for physical modeling and simulation of the selective laser sintering process was developed, validated and implemented in this paper. This model applies the essence of the traditional discrete element models for granular material but allows for changing the size of discrete particles based on the accuracy requirements of the problem. Numerical shape functions were developed by solving the steady state heat transfer problem at the particle level and their accuracy in performing temperature interpolations between larger nodes was demonstrated.

Using this physical modeling scheme, it is shown that thermal simulation of the SLS process can be performed with higher computational speeds compared to traditional DEM and without loss in simulation accuracy. This could open the door for predictive modeling and real time process control of the powder based additive manufacturing processes. 
The future steps of this research is intended to use results of this simulation tool to perform predictive analysis of the mechanical properties of SLS products. As the model developed in this paper is capable of predicting the microstructure of SLS products, methods could be developed to characterize mechanical properties of the products to complete the physical modeling of the SLS process. Moreover, parallelization could help improve the simulation runtime significantly.

The developed model, as well as all other simulation tools, uses a few simplifications in predicting the properties of powdered material, modeling the laser powder interaction, simulation of fusion between powder particles, and generating shape functions for multi-scale analysis. With the rising amount of research and industrial efforts in this area, there remains a need for a comprehensive study that compares the currently used models for simulation of powder based additive manufacturing in one setting and addresses their benefits and drawbacks. A general comparison of all the available simulation tools would be of significant importance to the field.

\section{Acknowledgments}

This work was performed as part of IRTG2057 on Physical Modeling for Virtual Manufacturing Systems and Processes as a collaborative effort between University of California at Davis and the University of Kaiserslautern, Germany.

\section{References}

[1] A. Aremu, I. Ashcroft, R. Wildman, R. Hague, C. Tuck, and D. Brackett, The effects of bidirectional evolutionary structural optimization parameters on an industrial designed component for additive manufacture, Proceedings of the Institution of Mechanical Engineers, Part B: Journal of Engineering Manufacture, 227 (2013), pp. 794--807.

[2] M. P. Bendsoe and O. Sigmund, Topology optimization: theory, methods, and applications, Springer Science \& Business Media, 2013.

[3] A. Chatterjee, S. Kumar, P. Saha, P. Mishra, and A. R. Choudhury, An experimental design approach to selective laser sintering of low carbon steel, Journal of Materials Processing Technology, 136 (2003), pp. 151--157.

[4] R. Dayal and T. Gambaryan-Roisman, Heat transfer in granular medium for application to selective laser melting: A numerical study, International Journal of Thermal Sciences, 113 (2017), pp. 38 -- 50.

[5] R. Ganeriwala and T. I. Zohdi, Multiphysics modeling and simulation of selective laser sintering manufacturing processes, Procedia CIRP, 14 (2014), pp. 299 -- 304.

[6] R. Ganeriwala and T. I. Zohdi, A coupled discrete element-finite difference model of selective laser sintering, Granular Matter, 18 (2016), p. 21.

[7] I. Gibson, D. Rosen, and B. Stucker, Additive manufacturing technologies: Rapid prototyping to direct digital manufacturing, Springer US, 2010. cited By 165.

[8] A. Gobal and B. Ravani, Physical modeling for selective laser sintering (sls) process, Journal of Computing and Information Science in Engineering, (2016).

[9] H. Haddad, M. Guessasma, and J. Fortin, Heat transfer by conduction using dem-fem coupling method, Computational Materials Science, 81 (2014), pp. 339 -- 347. 
[10] D. He, N. N. Ekere, and L. Cai, Computer simulation of random packing of unequal particles, Phys. Rev. E, 60 (1999), pp. 7098--7104.

[11] S. Kolossov, E. Boillat, R. Glardon, P. Fischer, and M. Locher, $3 d\{F E\}$ simulation for temperature evolution in the selective laser sintering process, International Journal of Machine Tools and Manufacture, 44 (2004), pp. 117 -- 123.

[12] I. Kovaleva, O. Kovalev, and I. Smurov, Model of heat and mass transfer in random packing layer of powder particles in selective laser melting, Physics Procedia, 56 (2014), pp. 400 -- 410.

[13] J. P. Kruth, P. Mercelis, J. V. Vaerenbergh, L. Froyen, and M. Rombouts, Binding mechanisms in selective laser sintering and selective laser melting, Rapid Prototyping Journal, 11 (2005), pp. 26--36.

[14] Y. Liang and X. Li, A new model for heat transfer through the contact network of randomly packed granular material, Applied Thermal Engineering, 73 (2014), pp. 984 -- 992.

[15] H.-T. Liao and J.-R. Shie, Optimization on selective laser sintering of metallic powder via design of experiments method, Rapid Prototyping Journal, 13 (2007), pp. 156--162.

[16] C. Martin, L. Schneider, L. Olmos, and D. Bouvard, Discrete element modeling of metallic powder sintering, Scripta Materialia, 55 (2006), pp. 425--428.

[17] D. Moser, S. Fish, J. Beaman, and J. Murthy, Multi-layer computational modeling of selective laser sintering processes, in ASME 2014 International Mechanical Engineering Congress and Exposition, American Society of Mechanical Engineers, 2014, pp. V02AT02A008-V02AT02A008.

[18] D. Moser, S. Pannala, and J. Murthy, Computation of effective radiative properties of powders for selective laser sintering simulations, JOM, 67 (2015), pp. 1194--1202.

[19] Computation of effective thermal conductivity of powders for selective laser sintering simulations, Journal of Heat Transfer, 138 (2016), p. 082002.

[20] J. M. Musser, Modeling of Heat Transfer and Reactive Chemistry for Particles in Gas-Solid Flow Utilizing Continuum-Discrete Methodology (CDM), West Virginia University Libraries, 2011.

[21] J. C. Nelson, S. Xue, J. W. Barlow, J. J. Beaman, H. L. Marcus, and D. L. Bourell, Model of the selective laser sintering of bisphenol-a polycarbonate, Industrial \& Engineering Chemistry Research, 32 (1993), pp. 2305--2317.

[22] D. Pal, N. Patil, K. Zeng, and B. Stucker, An integrated approach to additive manufacturing simulations using physics based, coupled multiscale process modeling, Journal of Manufacturing Science and Engineering, 136 (2014), pp. 061022--061022.

[23] J. Panetta, Q. Zhou, L. Malomo, N. Pietroni, P. Cignoni, and D. Zorin, Elastic textures for additive fabrication, ACM Transactions on Graphics, 34 (2015). cited By 14; Conference of ACM Special Interest Group on Computer Graphics and Interactive Techniques Conference, SIGGRAPH 2015 ; Conference Date: 9 August 2015 Through 13 August 2015; Conference Code: 114267.

[24] F. Parhami and R. McMeeking, A network model for initial stage sintering, Mechanics of Materials, 27 (1998), pp. 111 -- 124. 
[25] R. B. Patil and V. Yadava, Finite element analysis of temperature distribution in single metallic powder layer during metal laser sintering, International Journal of Machine Tools and Manufacture, 47 (2007), pp. 1069 -- 1080.

[26] S. Price, B. Cheng, J. Lydon, K. Cooper, and K. Chou, On process temperature in powder-bed electron beam additive manufacturing: process parameter effects, Journal of Manufacturing Science and Engineering, 136 (2014), p. 061019.

[27] J. Rojek, S. Nosewicz, K. Pietrzak, and M. Chmielewski, Simulation of powder sintering using a discrete element model, acta mechanica et automatica, 7 (2013), pp. 175--179.

[28] D. W. Rosen, Research supporting principles for design for additive manufacturing: This paper provides a comprehensive review on current design principles and strategies for am, Virtual and Physical Prototyping, 9 (2014), pp. 225--232.

[29] E. Rougier, A. Munjiza, and N. W. M. John, Numerical comparison of some explicit time integration schemes used in dem, fem/dem and molecular dynamics, Int. J. Numer. Meth. Engng., 61 (2004), pp. 856--879.

[30] J. C. Steuben, A. P. Iliopoulos, and J. G. Michopoulos, Discrete element modeling of particlebased additive manufacturing processes, Computer Methods in Applied Mechanics and Engineering, 305 (2016), pp. 537 -- 561.

[31] B. Vayre, F. Vignat, and F. Villeneuve, Designing for additive manufacturing, Procedia CIRP, 3 (2012), pp. 632--637.

[32] J. A. Williams and R. S. Dwyer-Joyce, Contact between solid surfaces, Modern Tribology Handbook, 1 (2001), pp. 121--162.

[33] J. Xing, W. Sun, and R. Rana, 3d modeling and testing of transient temperature in selective laser sintering (sls) process, Optik - International Journal for Light and Electron Optics, 124 (2013), pp. $301--304$.

[34] K. Zeng, D. Pal, N. Patil, and B. Stucker, A new dynamic mesh method applied to the simulation of selective laser melting, in Proceedings of the Solid Freeform Fabrication Symposium, Austin, TX, Aug, 2013, pp. 12--14.

[35] H. Zhang, Q. Zhou, and Y. Zheng, A multi-scale method for thermal conduction simulation in granular materials, Computational Materials Science, 50 (2011), pp. 2750 -- 2758.

[36] T. I. Zohdi, Rapid simulation of laser processing of discrete particulate materials, Archives of Computational Methods in Engineering, 20 (2013), pp. 309--325. 